\title{
Marco de trabajo de la función de tutoría universitaria para favorecer la permanencia estudiantil con enfoque cibersistémico transdisciplinario
}

\author{
A framework of the university tutoring function to promote student \\ permanence, with a transdisciplinary cyber systemic approach
}

Estrutura da função de tutoria universitária para promover a permanência do aluno com uma abordagem ciber-sistêmica transdisciplinar

Matilde Reséndiz-Castro

Instituto Politécnico Nacional, México mresendizc1700@alumno.ipn.mx https://orcid.org/0000-0001-5419-7652

Rosalba Zepeda-Bautista

Instituto Politécnico Nacional, México rzb0509@hotmail.com https://orcid.org/0000-0003-0988-8619

\section{Resumen}

El abandono estudiantil es un fenómeno socioeducativo que afecta a todos los niveles educativos. En América Latina se necesita identificar factores asociados a la permanencia estudiantil a través de diagnósticos que identifiquen áreas de oportunidad específicas de cada contexto educativo. Por ello, el objetivo de la investigación fue crear un marco de trabajo de la función de tutoría universitaria, fundamentado en un enfoque comunicativo, con modelos representativos de las necesidades de interacción del contexto educativo que posibiliten un programa y plan de trabajo que incidan en la atención y permanencia de los estudiantes. El marco de trabajo se aplicó en un estudio de caso bajo un enfoque cibersistémico transdisciplinario y de participación acción investigación. A nivel de intervención, se 


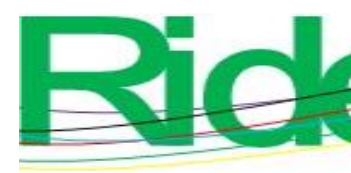

Revista Iberoamericana para la
Investigación y el Desarrollo Educativo
ISSN $2007-7467$

tomaron como base en indicadores de un diagnóstico de estudiantes-profesores y empresas en tres categorías: académica (criterios de evaluación, calificaciones parciales, instrumentos de evaluación y tiempo de estudio), personal (vocación, plan de carrera) y económica (becas internas, bolsa de trabajo). La población estuvo integrada por estudiantes de un programa de estudios de una universidad tecnológica en México que cursaban (entre 2018 y 2019) los dos primeros periodos cuatrimestrales de su carrera. Los resultados muestran un ejemplo aplicativo de un marco de trabajo integrado por: 1) la fundamentación cibersistémica transdisciplinaria de la función de tutoría universitaria, y 2) dos modelos: modelo de tutoría universitaria integral y modelo de gestión sistémico de la tutoría universitaria. A partir de estos se obtuvo: i) un programa institucional general de tutoría, y ii) su planeación de acción sistémica de tutoría con la clasificación de los indicadores, alineados con el modelo educativo estudio de caso y su modelo nacional de tutoría. Con la aplicación se obtuvo $76 \%$ de permanencia estudiantil. En el tercer y hasta el penúltimo cuatrimestre no se aplicó el programa, y se obtuvo como resultado una permanencia de $38 \%$. Se concluye, por tanto, que aun cuando la mayoría de las investigaciones indica que en promedio se pierde $50 \%$ de estudiantes en el primer ciclo estudiantil, se obtuvo $26 \%$ por arriba de lo esperado. Por tal motivo, se puede afirmar que es necesario un programa de atención estudiantil integral que se retroalimente y se ajuste a las necesidades de los estudiantes abarcando la cantidad de estados posibles de estos durante toda su trayectoria académica, pues se registra un riesgo de deserción de hasta $50 \%$ antes de la estadía industrial.

Palabras clave: Enseñanza y formación, industria y educación, ingeniería de sistemas, pertinencia de la educación, psicosociología de la educación.

\section{Abstract}

Student abandonment is a socio-educational phenomenon that affects all educational levels. In Latin America, it is to identify factors associated with student permanence through diagnoses that identify specific areas of opportunity in each educational context. To promote student permanence at the top level, the objective of this research was to create a framework for the university tutoring function. To this end, a case study was used under a cyber-systemic transdisciplinary approach to research participation. The population was a student of a curriculum at a Technological University in Mexico. The period of the investigation went from the last quarter of 2018 to the first quarter of 2019. Diagnostic indicators for students, 


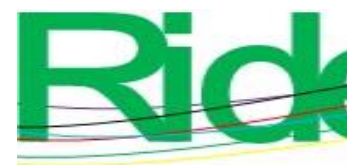

Revista Iberoamericana para la
Investigación y el Desarrollo Educativo
ISSN $2007-7467$

teachers and employers organized in three vulnerable areas were used: academic (evaluation criteria, partial qualifications, evaluation tools and study time), staff (vocation, career plan) and economic (internal scholarships, work bag). The results show an example of a framework composed of: (a) The transdisciplinary systemic cyber foundation of the university tutoring function; b) Two models: Integral University Tutoring Model and Systemic Management Model of University Tutoring. From the models was obtained c) General Institutional Tutoring Program and its d) Planning of Systemic Tutoring Action with the classification of indicators, aligned with the educational model case study and its national model of tutoring. Based on the information of the participating students during the first two semesters, a $76 \%$ student stay was quantified, with the application of the planning up to the second quarter. In the third and even the penultimate quarter, the program was not implemented, resulting in a $38 \%$ permanence. It is concluded that a student care program is needed that, in addition to its comprehensive implementation, is backed up and adjusted to the needs of students covering the number of possible student states.

Keywords: Teaching and training, industry and education, systems engineering, the relevance of education, psychosociology of education.

\section{Resumo}

A evasão escolar é um fenômeno socioeducativo que afeta todos os níveis educacionais. Na América Latina, é necessário identificar os fatores associados à retenção de alunos por meio de diagnósticos que identifiquem áreas de oportunidade específicas para cada contexto educacional. Portanto, o objetivo da pesquisa foi criar um referencial para a função de tutoria universitária, a partir de uma abordagem comunicativa, com modelos representativos das necessidades de interação do contexto educacional que possibilitem um programa e plano de trabalho que afetem a atenção e a permanência do alunos. $O$ framework foi aplicado em um estudo de caso sob uma abordagem cibersistêmica transdisciplinar e participação em pesquisa-ação. Ao nível da intervenção, tomaram-se como base indicadores de diagnóstico de alunos-professores e empresas em três categorias: académica (critérios de avaliação, notas parciais, instrumentos de avaliação e tempo de estudo), pessoal (vocação, plano de carreira) e económica (interna bolsas de estudo, banco de empregos). A população foi formada por alunos de um programa de estudos de uma universidade tecnológica no México que cursavam (entre 2018 e 2019) os dois primeiros quadrimestres de carreira. Os resultados mostram um 


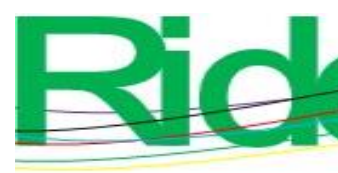

Revista Iberoamericana para la
Investigación y el Desarrollo Educativo
ISSN $2007-7467$

exemplo de aplicação de uma estrutura composta por 1) a base cibersistêmica transdisciplinar da função de tutoria universitária, e 2) dois modelos: o modelo de tutoria universitária abrangente e o modelo de gestão sistêmica da tutoria universitária. Destes, i) obteve-se um programa geral de tutoria institucional e ii) seu planejamento de ações de tutoria sistêmica com classificação de indicadores, alinhado ao modelo educacional de estudo de caso e seu modelo nacional de tutoria. Com o aplicativo, obteve-se $76 \%$ de permanência do aluno. No terceiro e até o penúltimo semestre o programa não foi aplicado, obtendo-se como resultado uma permanência de $38 \%$. Conclui-se, portanto, que embora a maioria das pesquisas indique que em média $50 \%$ dos alunos se perdem no primeiro ciclo, obteve-se $26 \%$ acima do esperado. Por isso, pode-se afirmar que é necessário um programa de atenção integral ao aluno que forneça feedback e se ajuste às necessidades dos alunos, englobando o número de estados possíveis destes ao longo de sua carreira acadêmica, pois há risco de evasão. a 50\% antes da parada industrial.

Palavras-chave: Educação e treinamento, indústria e educação, engenharia de sistemas, relevância da educação, psicossociologia da educação.

Fecha Recepción: Febrero 2021

Fecha Aceptación: Agosto 2021

\section{Introducción}

Existe vasta información sobre las actividades educativas que se realizan en las instituciones de educación superior (IES) para mejorar indicadores de rendimiento académico (RA), como la eficiencia terminal y la permanencia educativa. La mayoría de los estudios en América Latina de 1990 a 2016 muestran las relaciones entre las variables que intervienen en el abandono estudiantil, pero solo $5 \%$ se encuentra en un nivel de intervención, otro $5 \%$ se refiere a la evaluación y tan solo $11 \%$ se han dedicado a modificar la realidad de dicho fenómeno (Munizaga, Cifuentes y Beltrán, 2017). La transformación educativa implica una serie de mecanismos de conjunto, retroalimentación e interacción como sistema. De acuerdo con Cano (2001), el RA debe identificarse en componentes del sistema educativo; este concepto pone énfasis en el desenvolvimiento integral de la persona e implica recurrir al funcionamiento de los procesos de enseñanza y aprendizaje en sus diferentes ámbitos, lo que permitiría el conocimiento del estado educativo para mejorar áreas 


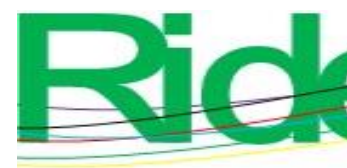

Revista Iberoamericana para la
Investigación y el Desarrollo Educativo
ISSN $2007-7467$

de oportunidad y ofrecer las condiciones para el desenvolvimiento integral de los alumnos y la optimización de los recursos.

De esta manera, el funcionamiento de la academia del sistema educativo en su eficacia debe hacer convergencia con la industria como un ecosistema (Ison, 2011), impactando con el flujo de talento y conocimientos para innovar a nivel local, regional y nacional (Ronde y Hussler, 2005) en una relación de colaboración universidad-industria (Hong, Zhu, Hou y Wang, 2019).

Aun cuando es clara la función y relevancia socioeconómica de la universidad, es contundente que la impartición de clases no es suficiente. Ante el incremento de la matrícula de estudiantes (Esteve, 2003), los modelos educativos deben diversificar formas y estrategias de retención colaborativa estudiantiles. Sin embargo, el reto va más allá, pues el fenómeno del abandono estudiantil se potencializa con la multicausalidad de los factores que lo afectan. De acuerdo con Torres, Acevedo y Gallo (2015), los componentes pueden ser personales, familiares y hasta culturales. Por su parte, Carvajal, Trejos y Gordillo (2017) indican que la práctica docente en el aula también puede ser una causa de la deserción.

Ante tal complejidad, esta investigación propone una alternativa con enfoque holístico y sistema abierto (Emery, 2004) con intervenciones de tipo transdisciplinaria transformacional (Wiek, Ness, Schweizer-Ries, Brand y Farioli, 2012) con base en la función de tutoría como una estrategia fundamental en la universidad (Reséndiz-Castro, 2019) para articular la comunicación y el acompañamiento de los estudiantes en la trayectoria académica (Romo, 2011). Esta, sustancialmente, se conforma de la interacción entre estudiantes, profesores y empleadores, pues sus funciones básicas son la retroalimentación (Reséndiz, 2021). La sistematización de la integración comunicativa como función principal ofrece la posibilidad de crear un aprendizaje organizacional y contribuir con objetivos institucionales. Destacar las relaciones entre estudiantes, profesores y empleadores en el sistema educativo permite distinguir los conceptos de aprendizaje que todo sistema debe extraer de su contexto 


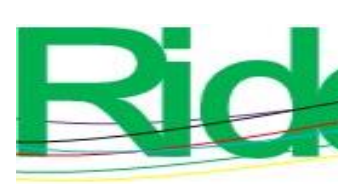

Revista Iberoamericana para la
Investigación y el Desarrollo Educativo
ISSN $2007-7467$

y usarlo como parte de la praxis en el contexto/mundo de la vida del alumno (Ison y Blackmore, 2014).

Por lo anterior, el objetivo de esta investigación es mostrar cómo con la creación de un marco de trabajo con enfoque cibersistémico transdisciplinario de la función de tutoría universitaria (MATCITTU) se favorece la permanencia académica de forma integral.

\section{Materiales y métodos}

Se utilizaron indicadores demográficos, socioeconómicos, pedagógicos, formativos, de equidad y de colaboración universidad-empresa de tres grupos de estudiantes: dos grupos de nuevo ingreso y uno de tercer cuatrimestre. Se usó la función de tutoría como estrategia de intervención en una universidad tecnológica (UT) en el nivel técnico superior universitario (TSU) en el Estado de México. El periodo de atención comprendió dos cuatrimestres de 2018 y 2019. La intervención concentró $64 \%$ de la matrícula total de estudiantes de un programa educativo como estudio de caso (Stake, 1998), de los cuales $21 \%$ fueron de nuevo ingreso. La figura 1 muestra la estructura del MATCITTU, integrado por: a) fundamentación de la función de tutoría universitaria con enfoque cibersistémico transdisciplinario (CST), b) Modelo de Tutoría Universitaria Integral (MOTUI) y Modelo de Gestión Sistémico de la Tutoría Universitaria (MOGESTU), alineados al Modelo Nacional de Tutoría para la Universidades Tecnológicas y Politécnicas de México (MONTU-UTyP) (Documento institucional elaborado por la Comisión de Rectores del Tema 21: Programa Nacional de Tutorías y Servicios de Apoyo al Estudiante, para las Universidades Tecnológicas y Politécnicas de México, 2017), c) un Programa Institucional General de Tutoría (PROIGET) y d) Planeación de Acción Sistémica de Tutoría (PAST) junto con un formato de calendario planificado de las actividades por desarrollar (FOCA).

La intervención fue de tipo psicosocial (Rodrigues, 1977) en combinación con ingeniería de sistemas (Checkland, 2001), de corte etnográfico (Hammersley y Atkinson, 2007), mediante la investigación-acción participación (IAP) (Colmenares, 2012). Se realizó una contrastación constante (Strauss y Corbin, 2002) entre los datos recabados y la realidad para elaborar una planeación interactiva (Ackoff, 2002) con enfoque transdisciplinario (Gibbs, 2017). Para la colección de datos se emplearon técnicas de observación participante, entrevistas focalizadas, grupos focales y documentos organizacionales (Hernández, Fernández y Baptista, 2007). 

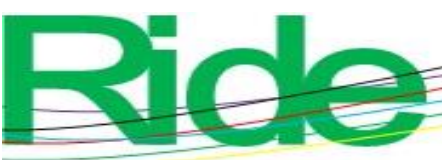

Revista Iberoamericana para la Investigación y el Desarrollo Educativo ISSN 2007 - 7467

Figura 2. Proceso de organización de los datos del marco de trabajo con enfoque cibersistémico transdisciplinario de la función de tutoría universitaria

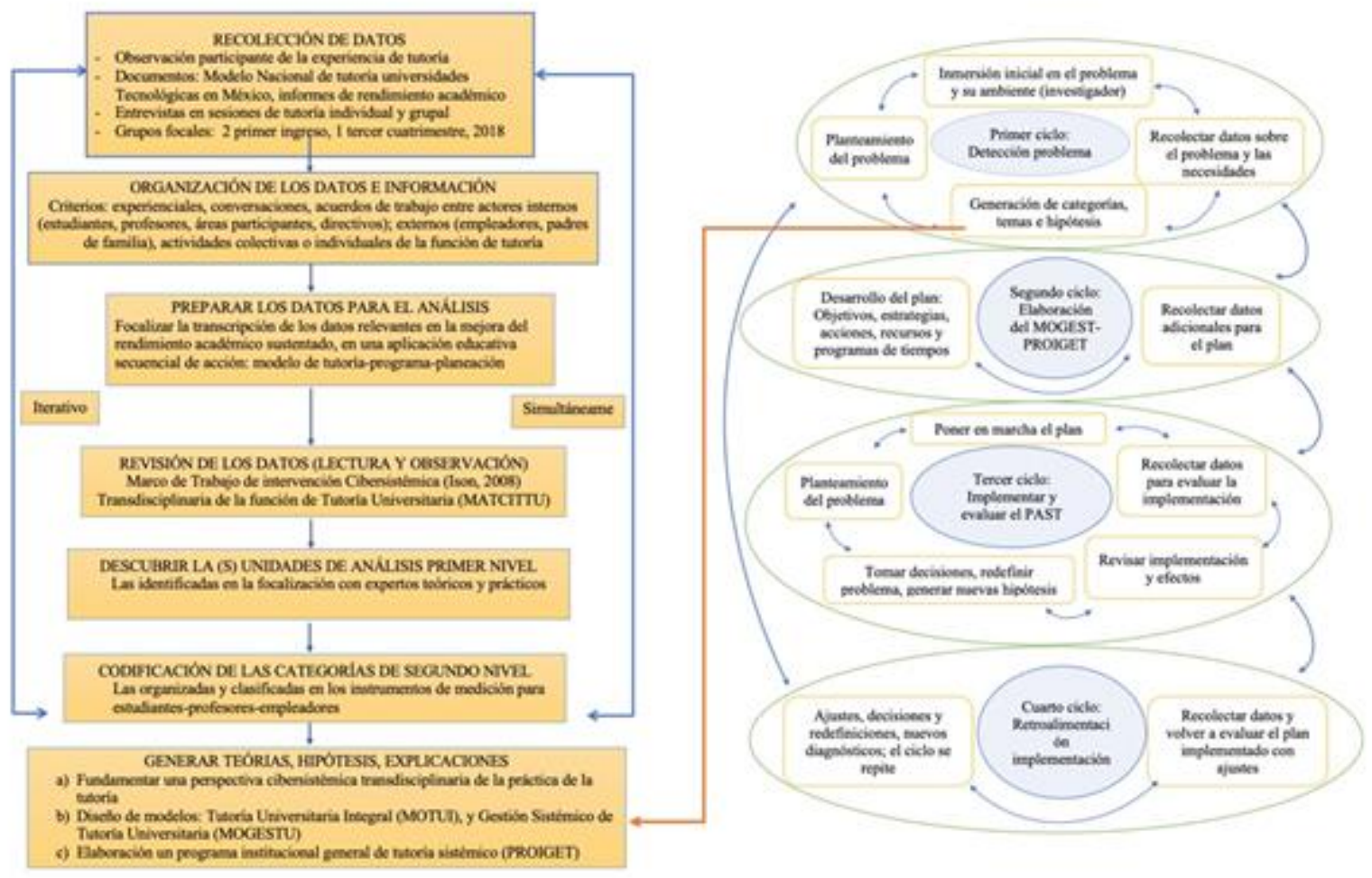

Fuente: Elaboración propia con base en Hernández et al. (2007)

\section{Resultados}

\section{Fundamentación de la función de tutoría universitaria con la perspectiva CST}

Antecedentes de la función de tutoría en una universidad tecnológica. La experiencia de colaboración docente (Glasserman, Reséndiz y Riquelme, 2010) y de integrar a estudiantes a niveles de trabajo en congresos, publicación y proyectos por un grupo de profesores (Reséndiz et al., 2013) sirvió de sustento para determinar de qué depende el desempeño de los estudiantes. La investigadora propuso al programa educativo un primer modelo de trabajo de tutoría. Se retomó la definición de tutoría que la Asociación Nacional de Universidades e Instituciones de Educación Superior (ANUIES) (2001) postuló como función principal en el acompañamiento y el apoyo docente basados en la atención personalizada para favorecer una mejor comprensión de los problemas que enfrenta el estudiante en su proceso de adaptación al medio universitario. 


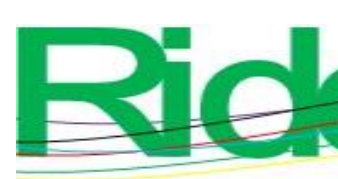

\section{Revista Iberoamericana para la Investigación y el Desarrollo Educativo ISSN 2007 - 7467}

También se consideraron aspectos individuales de cada estudiante para apoyar su desempeño y lograr sus objetivos académicos. Con ello se orientó la función de la tutoría, las áreas involucradas, los niveles de intervención y los recursos que se utilizaban para apoyar la permanencia de los estudiantes. La figura 3 muestra el modelo de tutoría propuesto por la investigadora en 2011, el cual venía funcionando desde 2008 y se utilizó en el rubro proceso de aprendizaje para la certificación del programa de estudios en 2010. El modelo muestra la población objetivo del tutor, las fuentes de información necesarias para su atención, las áreas de focalización (premisas de abandono escolar) y los recursos institucionales para atender los aspectos detectados como vulnerables.

Figura 3. Modelo de tutoría división académica de procesos de producción

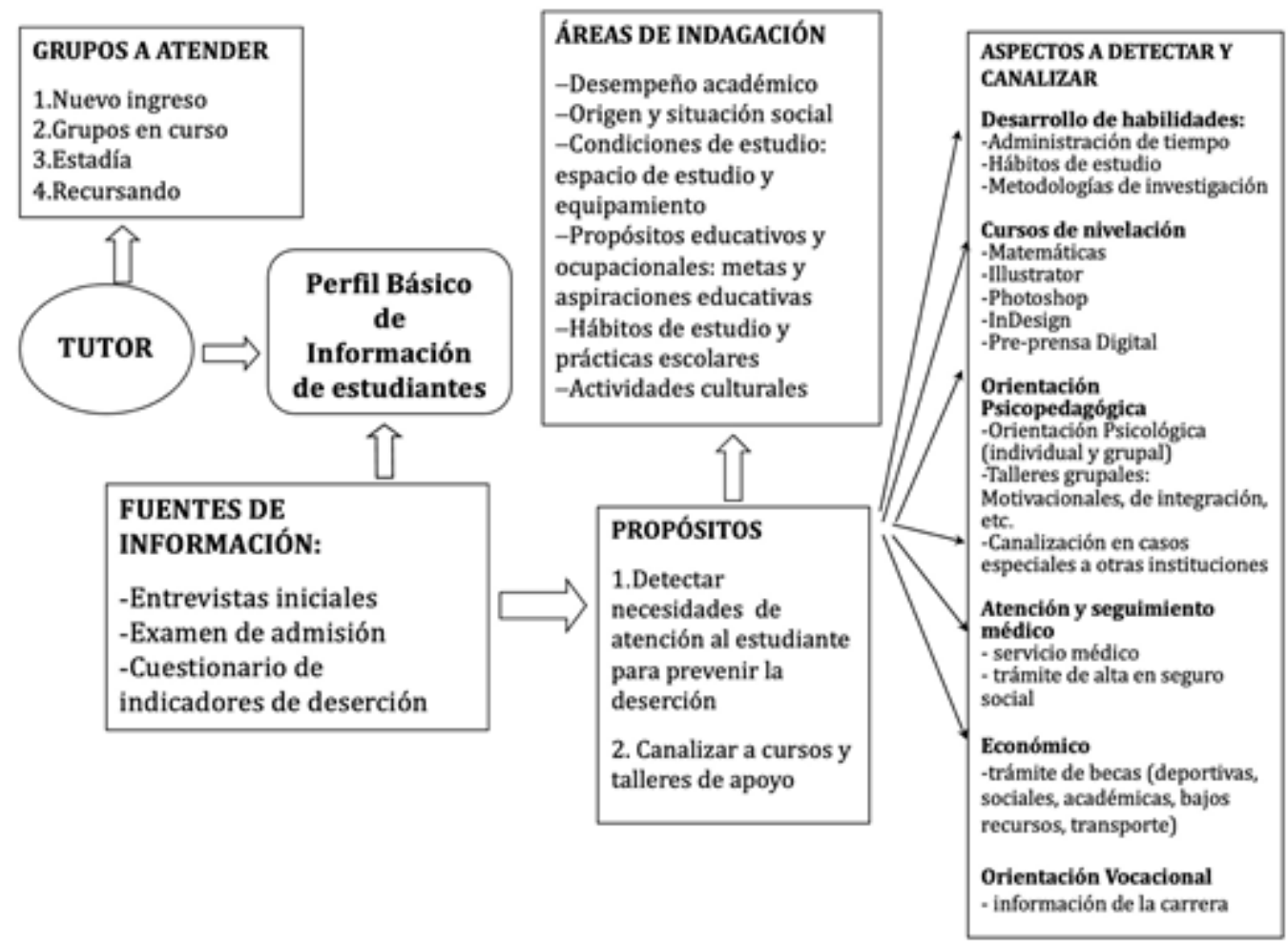

Fuente: Elaboración propia

Fue claro que la función de tutoría podría mejorar el desempeño de los estudiantes, pues los preparaba para su quehacer laboral. Sin embargo, aun cuando con la labor profesional como tutora se logró apoyar a algunos estudiantes, los indicadores de reprobación y deserción estudiantil continuaban, al mismo tiempo que no se lograba un método en el cual todos los tutores se basaran para atender a los estudiantes. 


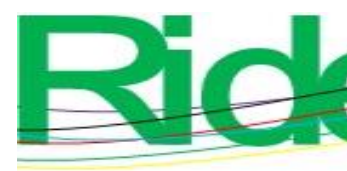

Revista Iberoamericana para la
Investigación y el Desarrollo Educativo
ISSN $2007-7467$

Con la experiencia de ejercer esta función, la investigadora inició una búsqueda para proponer de forma científica un método de atención en materia de rezago y deserción escolar para consolidar la formación profesional estudiantil de largo plazo. En 2017, con el Modelo Nacional de Tutoría para la Universidades Tecnológicas y Politécnicas de México (MONTUUTyP) (Documento institucional elaborado por la Comisión de Rectores del Tema 21: Programa Nacional de Tutorías y Servicios de Apoyo al Estudiante, para las Universidades Tecnológicas y Politécnicas de México, 2017), emitido por la Coordinación General de Universidades Tecnológicas y Politécnicas (CGUTyP), se realizó un intento de homogenizar la función de tutoría en el subsistema de UT para favorecer la permanencia y la eficiencia terminal, medibles a través de indicadores de control y seguimiento con base a los estándares del Modelo Educativo de Universidades Tecnológicas.

En el MONTU-UTyP se explica que la tutoría es un proceso de acompañamiento personalizado por parte de docentes y especialistas "durante la formación de estudiantes, que implementa estrategias de detección, orientación, canalización y seguimiento en los ámbitos académico, socioeconómico y personal (...) con el fin de coadyuvar en la formación integral y el logro de los objetivos institucionales" (MONTU-UTyP, p. 10). Además, refiere que deberán autoevaluar el desempeño de los estudiantes tutorados. La modalidad de atención puede ser individual, grupal o de pares, así como presencial o virtual. Estos antecedentes sirvieron para realizar la fundamentación teórica del enfoque CST y la función de tutoría.

Cibersistémica transdisciplinaria de la tutoría. Con una ponencia de la autora en el 8. ${ }^{\circ}$ Encuentro Nacional de Tutoría organizado por la Asociación Nacional de Universidades e Instituciones de Educación Superior (ANUIES) en coordinación con la Universidad Autónoma de San Luis Potosí (UASLP) en 2018, se enfatizó la capacidad comunicativa de la tutoría para mejorar el RA de los estudiantes (Reséndiz-Castro, 2019). Gradualmente, el enfoque se convirtió en una aproximación metodológica de intervención en el programa del doctorado de Ingeniería de Sistemas de la ESIME Zacatenco del IPN (Reséndiz-Castro, Peón-Escalante y Zepeda- Bautista, 2018). La fundamentación se plantó en el marco del MONTU-UTyP, que tiene como objetivo elaborar un programa institucional de tutorías, hasta la fecha inexistente en la universidad caso de estudio. 


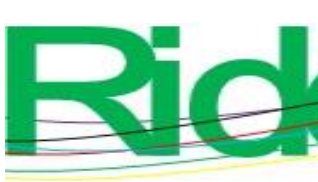

Revista Iberoamericana para la Investigación y el Desarrollo Educativo ISSN $2007-7467$

Figura 5. Modelo de Gestión de la Tutoría Universitaria MOGESTU

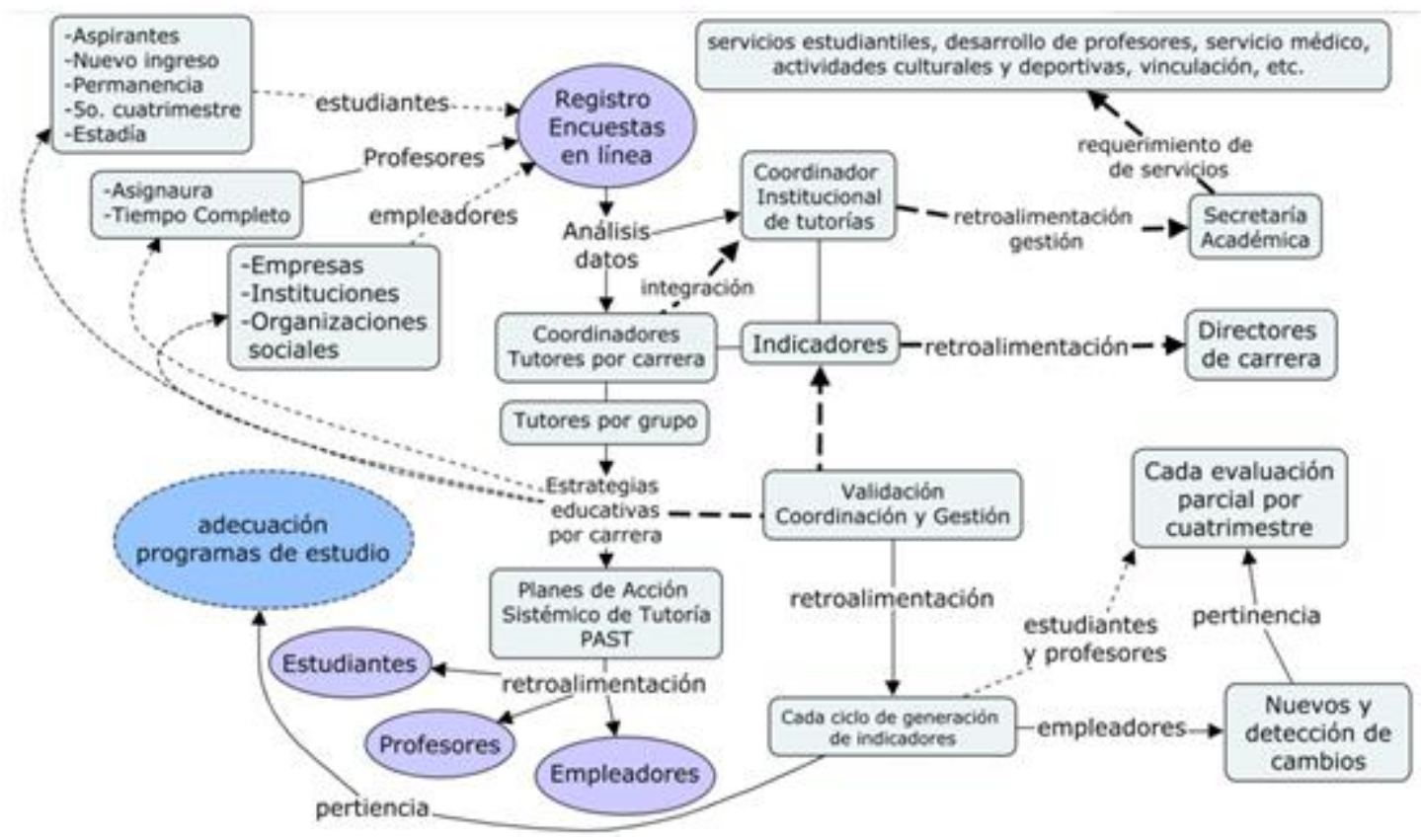

Fuente: Elaboración propia con base en el modelo de Gómez (UNAM, titular del taller de diseño de un PAT en el 8. Encuentro Nacional de Tutorías ANUIES-UASLP, 2018) y el

Modelo Nacional de Tutoría (Universidades Tecnológicas y Politécnicas de México de 2017)

Para evaluar las implementaciones de la función de la tutoría se propone que la retroalimentación tenga una periodicidad tipo holónica (Wilber, 2011) por cada corte parcial de evaluación. Esto permite hacer los ajustes necesarios durante la permanencia de los estudiantes, por lo menos, tres veces en cada cuatrimestre y entre cada cuatrimestre durante al menos un año para obtener resultados y retroalimentación del impacto de las estrategias implementadas. La gestión se encuentra alineada al MONTU-UTyP, por lo que incorpora a directores de carrera y las funciones de la Secretaría Académica para la gestión de requerimientos de servicios estudiantiles específicos con cada PAT y en general con un programa institucional de atención al estudiante. Una vez propuestos los modelos, se sugiere un ejemplo de programa institucional.

\section{Programa Institucional General de Tutoría (PROIGET)}

La tabla 1 muestra la descripción estructural de los aspectos del PROIGET con funciones, acciones e interacciones de los diferentes niveles de organización institucional. 


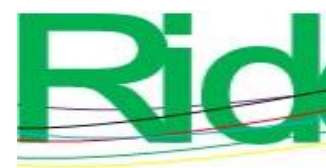

Revista Iberoamericana para la Investigación y el Desarrollo Educativo ISSN 2007 - 7467

Tabla 1. Descripción de aspectos de un Programa Institucional General de Tutoría

(PROIGET)

\begin{tabular}{|l|l|}
\hline \multicolumn{1}{|c|}{ Aspectos } & \multicolumn{1}{|c|}{ Descripción } \\
\hline $\begin{array}{l}\text { Situación función de tutoría } \\
\text { por división académica }\end{array}$ & $\begin{array}{l}\text { Identificación del estado actual de la función de tutoría, } \\
\text { utilidad, niveles, necesidades de intervención y priorización } \\
\text { de indicadores de diagnóstico de RA de estudiantes, } \\
\text { profesores y empleadores. }\end{array}$ \\
\hline $\begin{array}{l}\text { Definición consensada de la } \\
\text { función de tutoría }\end{array}$ & $\begin{array}{l}\text { Definición consensada de la función de tutoría, alineada con } \\
\text { modelo educativo, modelo nacional de tutoría u } \\
\text { organizaciones educativas como la ANUIES. }\end{array}$ \\
\hline $\begin{array}{l}\text { Acciones de atención al } \\
\text { alumnado por grupo } \\
\text { vulnerable }\end{array}$ & $\begin{array}{l}\text { Con base en indicadores, clasificar a los estudiantes por } \\
\text { atender, acciones por nivel de intervención y clasificación } \\
\text { vulnerable. }\end{array}$ \\
\hline $\begin{array}{l}\text { Actividades sugeridas por } \\
\text { grupo vulnerable } \\
\text { (estudiantes) }\end{array}$ & $\begin{array}{l}\text { Por clasificación vulnerable: Personal, académica y } \\
\text { económica; se identifican y describen las actividades para } \\
\text { planificar. }\end{array}$ \\
\hline $\begin{array}{l}\text { Actividades de } \\
\text { reforzamiento (profesores) }\end{array}$ & $\begin{array}{l}\text { Actividades para profesores basadas en las necesidades } \\
\text { detectadas de servicio al estudiante. }\end{array}$ \\
\hline Seguimiento del programa & Mecanismos para el seguimiento al programa. \\
\hline
\end{tabular}

Fuente: Elaboración propia

Una vez implementado, la retroalimentación iterativa (cibersistémica) permitiría realizar las adecuaciones necesarias para conseguir los objetivos para cada área de interacción (División Académica) en diferentes niveles de la organización (servicios al estudiante). A continuación, se presenta un ejemplo sugerido de cada aspecto integrante de un PROIGET.

Situación actual de la función de tutoría. La figura 6 muestra un ejemplo de focalización de las funciones del tutor aplicado al estudio de caso con respecto a la detección de necesidades de la población por atender. 


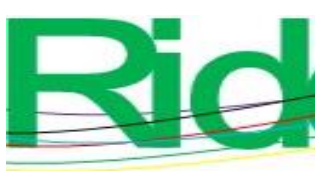

Revista Iberoamericana para la Investigación y el Desarrollo Educativo ISSN $2007-7467$

Figura 6. Ejemplo de focalización de las funciones del tutor en la universidad

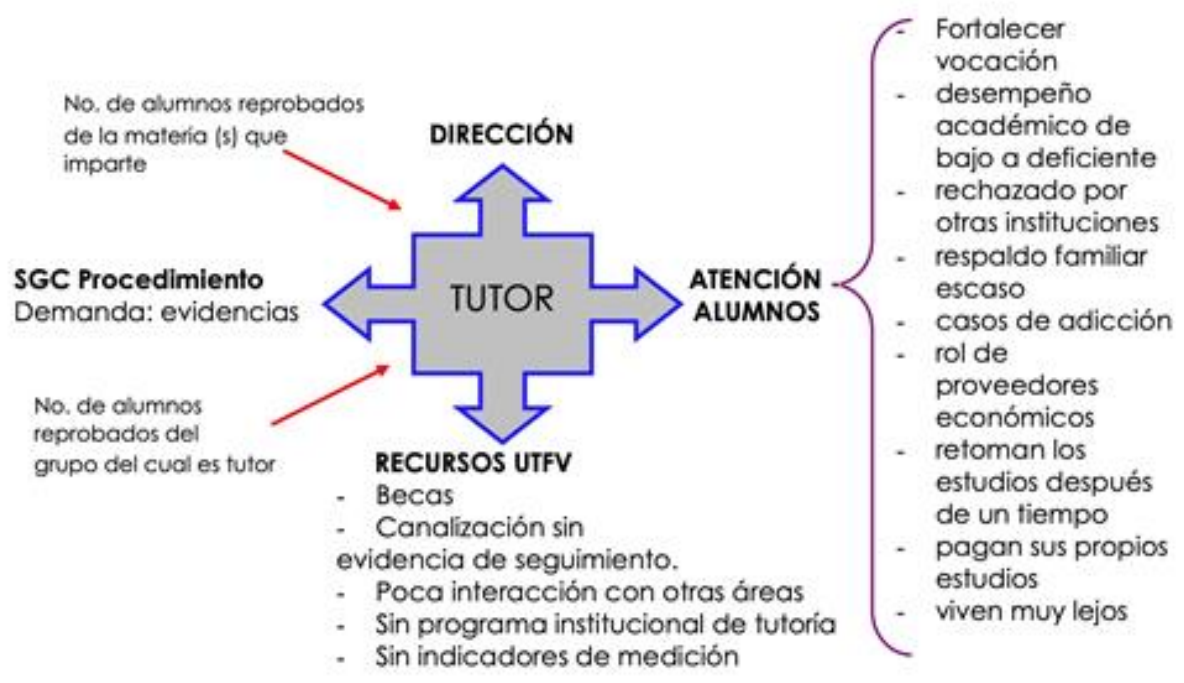

Fuente: Elaboración propia

Definición de la tutoría. Basado en la propuesta de la ANUIES (2001), se tiene que la tutoría es un proceso de acompañamiento durante la formación de los estudiantes que se concreta mediante la atención personalizada a un alumno o a un grupo reducido de alumnos por parte de académicos competentes y formados para esta función, los cuales se sustentan en las teorías del aprendizaje más que en las de la enseñanza. El establecimiento de un proceso de tutoría responde a un conjunto de objetivos relacionados con la integración formativa del alumnado, la retroalimentación del proceso educativo, la motivación del estudiante, el desarrollo de habilidades para el estudio y el trabajo, el apoyo académico y la orientación, y sobre todo con el perfil de egreso.

Acciones de atención al alumnado por grupo vulnerable. La tabla 2 muestra algunas sugerencias de clasificación de estudiantes por tipo de grupo (nivel de intervención) en correspondencia con las acciones posibles para implementar por grupo vulnerable. 


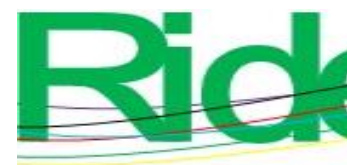

Revista Iberoamericana para la
Investigación y el Desarrollo Educativo
ISSN $2007-7467$

especiales para mantener y retroalimentar los conocimientos concernientes a las diferentes profesiones, animando y fortaleciendo el deseo por el estudio, así como su continuidad.

Para el área económica se sugieren las siguientes actividades: 1) servicios estudiantiles. En coordinación con el área correspondiente, se debe conocer la proyección de los estudiantes que requieren apoyos económicos mediante la condonación de inscripción, otorgamiento de becas internas o externas, etc.; 2) bolsa de trabajo. En coordinación con el área correspondiente, actualizar y ampliar los requerimientos de capital humano interno y externo (municipios aledaños, organizaciones gubernamentales, comercios y empresas locales y regionales).

Actividades de reforzamiento (profesores). Con base en un diagnóstico del personal docente, valorar la factibilidad de subsanar las debilidades de formación con respecto a las necesidades detectadas en el diagnóstico de estudiantes. Dependiendo de ello, se sugieren las siguientes actividades generales que no deben suplir a un programa de capacitación: 1) proyectos interdisciplinarios (profesores coordinadores). Definición de proyectos interdisciplinarios para los grupos; 2) programa de capacitación y actualización docente. Se genera a partir de las necesidades de congruencia académica con los conocimientos de diferentes profesiones para realizar las actividades prácticas propias de la carrera y pertinentes con el perfil del egresado. Esto lleva como consecuencia definir academias; 3) reconocimiento institucional de academias. Reunión de grupos de profesores por áreas de conocimiento para revisar los programas de estudio con el fin de promover la congruencia de los conocimientos vigentes con el currículo. Generar manuales de asignatura que permitan definir las prácticas correspondientes y congruentes con los planes y programas de estudio; 4) proyectos industriales. Reunión de profesores de tiempo completo para definir los proyectos y acordar la dinámica de seguimiento de proyectos con las empresas; 5) proyectos de servicio social. Reunión de profesores para definir la dinámica para determinar proyectos de servicio social, duración, objetivos, etc.; 6) círculos de tutoría. Se deben hacer reuniones con los profesores tutores para compartir experiencias de tutoría, definir planes de acción para resolver las dificultades que obstaculizan el desempeño y permanencia de los alumnos, así como la retroalimentación de las necesidades pedagógicas que ayuden y contribuyan a resolver problemas académicos con los alumnos. 


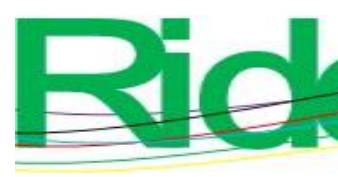

Revista Iberoamericana para la
Investigación y el Desarrollo Educativo
ISSN $2007-7467$

Seguimiento del programa de tutoría. Mediante el reporte del programa (físico o informático) se da seguimiento a las acciones cuatrimestrales planeadas (por acción, alumno, grupo y tutor). La información y experiencia de tutoría servirá para trabajar en los círculos de tutoría para profesores, y así reforzar y tomar acciones en relación con las necesidades de los grupos vulnerables.

Hasta aquí se muestra de forma general un ejemplo de programa institucional de tutoría para su implementación en cualquier IES, el cual debe adecuarse al modelo educativo y alinearse con el modelo de tutoría indicado por cada organización, así como considerar la normatividad interna vigente. Una vez integrado el PROIGET, se sugiere realizar una planificación interactiva (Ackoff, 2002), es decir, en conjunto con los estudiantes y avalado por directores, profesores y, en su caso, por las instancias que intervendrán. A continuación, se describe un ejemplo de implementación de un PAST del caso de estudio.

\section{Planeación de Acción Sistémico de Tutoría (PAST) a partir de indicadores}

\section{diagnósticos}

El PAST es una fase del PROIGET enfocada en el desempeño de los estudiantes en aula. La acción se organizó con la información obtenida del diagnóstico y se retroalimentó con las necesidades y calificaciones posteriormente recabadas de los estudiantes. Se hizo una triangulación de la información recabada a partir de instrumentos para el diagnóstico aplicados mediante entrevistas a estudiantes con dificultades académicas y a las evidencias de trabajo académico de la dirección de la carrera estudio de caso. Cabe aclarar que en el caso de estudio no existe evidencia académica sistemática y consistente del proceso de evaluación por parte de los profesores que refieran estrategias educativas (basadas en diagnósticos, indicadores de desempeño, etc.), características de instrumentos de evaluación (de forma individual se define la forma de evaluar, no precisamente con instrumentos de evaluación), entrega de programas académicos a los estudiantes, y verificación de que se realicen. Si algún estudiante refiere inconformidad en sus evaluaciones, la dirección solicita al profesor en cuestión documentar el proceso de evaluación para determinar si existe alguna inconsistencia.

En la tabla 4 se describen algunos indicadores de diagnóstico con los que se integra el PAST en cada grupo de estudio indicado por el MONTU-UTyP, clasificados en categorías de tipo académicas, personales y económicas. Con una hora a la semana integrada al horario 


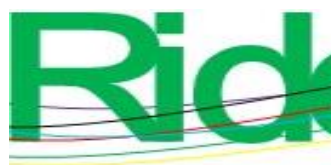

Revista Iberoamericana para la Investigación y el Desarrollo Educativo ISSN 2007 - 7467

de los estudiantes y con la retroalimentación de estos, se sugirió el uso de un formato para registrar las actividades de los estudiantes participantes por categoría.

Tabla 4. Integración de indicadores clasificados por grupo vulnerable

\begin{tabular}{|l|l|l|}
\hline \multicolumn{1}{|c|}{ Académica } & \multicolumn{1}{c|}{ Personal } & \multicolumn{1}{c|}{ Económica } \\
\hline Criterios de avaluación. & Vocación. & $\begin{array}{l}\text { Becas internas y } \\
\text { externas. }\end{array}$ \\
\hline $\begin{array}{l}\text { Calificaciones parciales o } \\
\text { continuas. }\end{array}$ & Plan de carrera. & Bolsa de trabajo. \\
\hline Instrumentos de evaluación. & & \\
\hline Tiempo de estudio. & & \\
\hline
\end{tabular}

Fuente: Elaboración propia

En la figura 7 se muestra el Formato de Calendario de Actividades (FOCA) organizado para los tres grupos de tutoría, previa sesión de sensibilización acerca de la importancia del acompañamiento y la función de la tutoría universitaria.

Figura 7. Formato calendario de actividades por grupo y programa educativo

\begin{tabular}{|c|c|c|c|c|}
\hline \multicolumn{3}{|c|}{ Programa: Nombre del programa educativo. } & \multicolumn{2}{|c|}{ Hoja ___ de } \\
\hline \multicolumn{5}{|l|}{ Tutor: Nombre del tutor. } \\
\hline Dimensión: Académica. & \multicolumn{2}{|c|}{ Grupo: Nombre del grupo } & \multicolumn{2}{|c|}{ Total: Alumnos inscritos } \\
\hline \multicolumn{5}{|c|}{ Actividades/fecha } \\
\hline Elaboración de CV & Fecha & \multicolumn{2}{|c|}{ Visita industrial } & Fecha \\
\hline \multicolumn{2}{|c|}{ Redactar objetivo de la actividad } & \multicolumn{3}{|c|}{ Redactar objetivo de la actividad } \\
\hline \multicolumn{5}{|c|}{ Registro de estudiantes por actividad } \\
\hline \multicolumn{2}{|c|}{ Nombre y firma de cada estudiante... } & \multicolumn{3}{|c|}{ Nombre y firma de cada estudiante... } \\
\hline
\end{tabular}

Fuente: Elaboración propia

El FOCA es una herramienta de trabajo con la que el profesor planea actividades y asistencias a partir de la detección de indicadores y necesidades de los estudiantes. Se integró con la información del diagnóstico y con el contraste de la experiencia de interacción en aula y con lo que expresan los estudiantes. Esta planificación debe llegar a un punto de acuerdo entre el profesor y estudiantes; con ello se organiza el registro de asistencias y la aceptación de cada actividad. Para tener claridad acerca de la implementación de cada actividad, se sugiere que cada una tenga un objetivo. Al FOCA creado se adjunta la documentación de las actividades de gestión para lograr los objetivos de cada actividad. Por ejemplo, para el grupo de $5 .^{\circ}$ cuatrimestre se presentó la lista de empresas vinculadas, un proyecto de estadía 


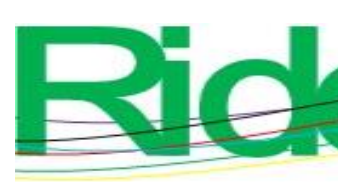

Revista Iberoamericana para la
Investigación y el Desarrollo Educativo
ISSN $2007-7467$

realizado en un cuatrimestre anterior, los datos de la empresa y se compartieron experiencias de trabajo del tutor con los empleadores.

Como recomendación general de aplicación de este MATCITTU, se deben realizar diagnósticos iterativos de acuerdo con el calendario escolar o evaluaciones parciales. Por cada diagnóstico se debe generar un PAST y tendrá una temporalidad de aplicación del periodo de evaluación que corresponda. Los PAST deben ajustarse a los indicadores generados en los diagnósticos realizados, de manera sucesiva y progresiva con estudiantes de nuevo ingreso y durante su permanencia (Reséndiz-Castro, 2021).

\section{Discusión}

La presente investigación se ubica en el campo educativo en general y en el de la educación superior en particular. El interés parte de detectar la deserción estudiantil como detonante del rezago académico y la implicación de la función de docente y de tutoría de la investigadora (Devereux, 2003). Poco después, la ANUIES (2001) define líneas de acción con respecto a lo que consideró una innovación educativa: la tutoría. En 2020, una política más apoya esta función: el marco de referencia del Consejo de Acreditación de la Enseñanza de la Ingeniería A. C. (CACEI), que incluye la valoración de índices de rendimiento escolar, los programas institucionales (asesoría y tutoría), las estrategias que apoyan el desempeño (trayectoria escolar) y la retención y permanencia de estudiantes (CACEI, 2020). Sin obligatoriedad de las IES para certificarse y para adoptar un programa de permanencia estudiantil, estas tienen diversas formas de atención de tutoría. Por ello, este estudio es pertinente y útil para sistematizar y transformar el RA del sistema, sin burocracia, incluyendo la participación de la comunidad educativa para evaluar competencias (Martínez, Tobón y Romero, 2017).

La relación del contexto de tutoría y su fundamentación CST potencializa su función y focaliza recursos organizacionales de cada institución. En esta relación la tutoría es un eje integrador entre estudiantes, profesores, organización y campo laboral (Reséndiz-Castro, 2019). Con ello, se asume que la universidad tiene el reto de diversificar la formación educativa, a partir de los cambios y necesidades que la sociedad requiere para contribuir con el equilibrio en la calidad de vida (Rama, 2006). Los modelos MOTUI y MOGESICT representan la relación del contexto como guías de intervención; en ellos se muestra la relevancia de la actividad docente como elemento primordial de cualquier sistema educativo 


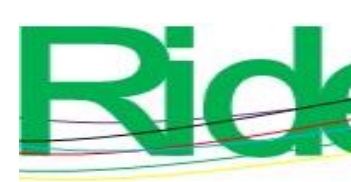

Revista Iberoamericana para la
Investigación y el Desarrollo Educativo
ISSN $2007-7467$

(Said-Hung, 2017). Como sistema abierto (Emery, 2004), la incorporación del ámbito laboral representado por el sector industrial permite construir cierta relación sustentable y pertinente, que de acuerdo con Tobón (2013) da sustento a la innovación de la socioformación técnica y profesional para establecer un contexto equilibradamente medible a nivel local, regional y nacional. Para Güemes-Castorena y Ponce-Jaramillo (2019) la simbiosis entre universidadempresa de colaboración genera autopoiesis para la investigación y desarrollo (I+D) y su vinculación interinstitucional incrementa las capacidades en ciencia y tecnología necesarias para la innovación social.

La relevancia de este marco de trabajo radica en la forma de sistematizar la permanencia estudiantil, lo que se puede constatar con el trabajo de Singh y Maloney (2019), en donde mediante mediciones, además de optimizar los recursos empleados para formar capital humano, se incentiva el desempeño laboral. Esto incluye al deporte en su relación con el RA. Al respecto, Gracia (2020) muestra una relación para la permanencia universitaria, identificada entre el autoconcepto físico y el RA, donde el autoconcepto sirve de andamio para la integración estudiantil (McGhie, 2017). De igual forma, Valencia-Peris-Valencia, Devís-Devís y Peiró-Velert (2016) enseñan cómo el conocimiento de diversas actividades sedentarias de los estudiantes se asocia con su efecto en el éxito académico.

La Planeación de Acción Sistémico de Tutoría (PAST) sirve de poco a la transformación de la realidad de los estudiantes (Wiek et al. 2012) si no se cuenta con mecanismos de diagnóstico que sistematicen la información del sistema. En la clasificación de variables del MONTU-UTyP, si bien son congruentes con la literatura, los indicadores deber precisarse según la operacionalización en cada institución. En este estudio fue preciso identificar la importancia operativa de cada indicador del área académica, con lo cual se englobaron aspectos claves del proceso de enseñanza-aprendizaje. Para el caso de criterios de evaluación, son concebidos como un conjunto de aprendizajes que los estudiantes deben alcanzar al finalizar un periodo de trabajo (Zabalza y Lodeiro, 2019), lo cual compete a la práctica docente. Con lo anterior se definen calificaciones parciales o continuas, así como instrumentos para evaluar el aprendizaje en cada periodo. Para Carvajal et al. (2017) la práctica docente es un soporte estudiantil poco indagado, a pesar de considerarse como causante del abandono estudiantil. Otro indicador del diagnóstico fue el desconocimiento de normas, procesos y reglamentos institucionales como marco de la práctica docente (Bayona, Oliveros y Saldarriaga, 2019), así como la práctica de rendición de cuentas, reflejada en 


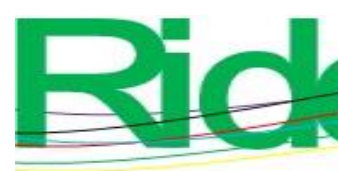

Revista Iberoamericana para la
Investigación y el Desarrollo Educativo
ISSN $2007-7467$

evaluaciones sin evidencia clara por parte de docentes. La organización de contenidos académicos (Rochin, 2021) y de evaluación son imprescindibles para estimar el tiempo de estudio, incluso los recursos por utilizar.

En cuanto al criterio personal, engloba aspectos como la vocación de la carrera elegida relacionada con el RA de los estudiantes (Carvajal y Trejos, 2016) y con los intereses de los estudiantes. Zumárraga et al. (2017) han demostrado que la vocación tiene un impacto modesto en los primeros niveles de estudio y se intensifica en el caso de algunas carreras. Con respecto a la variable económica, existe evidencia de la necesidad de trabajar de los estudiantes (Fleet y González, 2017). En otros casos, es un factor de abandono derivado del tiempo dedicado a estudiar (Herrero, Escanés y Ayllón, 2017).

Como se puede inferir, la ausencia de mecanismos para constatar la gestión de la permanencia estudiantil en un sentido de intervención no debe ser asumida como dada, sino como compleja (Tobón, 2013). El monitoreo de indicadores educativos no es un tema nuevo, pero sí su sistematización. Esta es un área de oportunidad para la identificación, seguimiento y medición en cursos en línea (Arnold y Campbell, 2013) y para predecir la deserción estudiantil (Tempelaar, Rienties, Mittelmeier y Nguyen, 2018), que también pueden ser aplicables a modalidades mixtas (en línea y presenciales) (Xing, Chen, Stein, y Marcinkowski, 2016).

\section{Conclusiones}

El marco de trabajo estratégico de intervención psicosocial de la función de tutoría universitaria con enfoque cibersistémico transdisciplinario se basó en indicadores de diagnóstico de estudiantes, profesores y empleadores para favorecer la permanencia de los alumnos. La función de la tutoría universitaria ha representado, desde hace un par de décadas, una unidad de interconexión entre la permanencia del estudiante y el contexto del sistema educativo. Los programas deben contemplar una base metodológica mínima basada en lo siguiente: a) una fundamentación de la tutoría universitaria, b) modelos de tutoría universitaria integral y de gestión sistémica, c) un programa institucional general de tutoría y d) una planeación de acción respaldada por su evidencia, así como su aplicación, evaluación y retroalimentación a través del tiempo. 

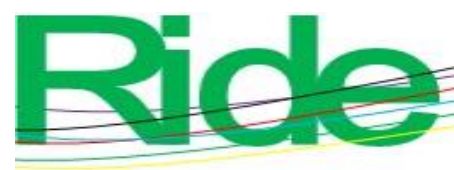

Revista Iberoamericana para la Investigación y el Desarrollo Educativo ISSN $2007-7467$

En tal sentido, y con la aplicación del Plan de Acción Sistémico de Tutoría (PAST) se observó que, para el cuatrimestre siguiente, el porcentaje de permanencia estudiantil de los grupos de nuevo ingreso fue de $76.56 \%$, es decir, hubo una retención de más de la mitad de dichos alumnos. Sin embargo, en los siguientes cuatrimestres (tercero, cuarto y quinto) y en el inicio del penúltimo (antes de la estadía) no se aplicó el programa, por lo que se observó una retención de $33 \%$, lo que significa que se perdió más de la mitad de los estudiantes. La permanencia estudiantil universitaria requiere - además de un programa institucional de tutoría, seguimiento, monitoreo y retroalimentación - la disposición de las autoridades de cada IES para focalizar la toma de decisiones y sus recursos organizacionales. Además, un sistema exige definir los elementos y funciones de cada uno de ellos de forma armónica y coherente, así como sus interacciones entre ellos y hacia afuera del sistema en una frontera definida. La implementación del marco de trabajo MATCITTU implica un compromiso entre autoridades, profesores y empleadores de cada IES. Es necesario identificar las necesidades de los estudiantes, la sinergia de trabajo organizacional y, sobre todo, resarcir áreas de oportunidad académica a cargo de los directivos. Los profesores, por ende, deben atender de manera permanente y focalizada a los estudiantes en sus avances y dificultades para alcanzar la formación de un profesional competente y comprometido con la sociedad.

\section{Futuras líneas de investigación}

Como trabajo futuro se puede profundizar en sistematizar el diagnóstico y su implementación, lo cual resultaría muy útil para generar perfiles educativos y crear un conjunto de estrategias educativas que permitan encarar con éxito el reto de la permanencia estudiantil. 


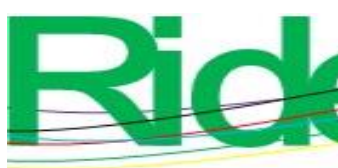
Revista Iberoamericana para la
Investigación y el Desarrollo Educativo
ISSN $2007-7467$

\section{Referencias}

Ackoff, R. L. (2002). El paradigma de Ackoff: una administración sistémica. México: Limusa.

Arnold, K. and Campbell, J. (2013). System and method of using academic analytics of institutional data to improve student success. Retrieved from https://patentimages.storage.googleapis.com/8a/e4/9c/9e8b53b004a146/US8412736 .pdf

Asociación Nacional de Universidades e Instituciones de Educación Superior [ANUIES] (2001). La tutoría como una estrategia viable de mejoramiento de la calidad de la educación superior. Una propuesta de la ANUIES para su organización y funcionamiento en las instituciones de educación superior (2. ${ }^{a}$ ed.). México: ANUIES. Recuperado de http://www.uimqroo.edu.mx/Documentos/Tutorias/MANUALTUTORIAS-

ANUIES.pdf

Bayona, L., Oliveros, S. y Saldarriaga, J. (2019). Conocimiento, interpretación y uso de las normas como forma de disminuir la deserción. Congresos CLABES. Recuperado de https://revistas.utp.ac.pa/index.php/clabes/article/view/2675.

Cano, S. S. J. (2001). Rendimiento escolar y sus contextos. Revista Complutense de Educación, 12(1), 15-80. Recuperado de https://revistas.ucm.es/index.php/RCED/article/view/RCED0101120015A/16836

Carvajal, O. P. y Trejos C. Á. (2016). Revisión de estudios sobre deserción estudiantil en educación superior en Latinoamérica bajo la perspectiva de Pierre Bourdieu. Congresos RLABES. Recuperado de https://revistas.utp.ac.pa/index.php/clabes/article/view/1324

Carvajal, O. P., Trejos C. Á. A. y Gordillo, M. S. (2017). La práctica docente en la Universidad Tecnológica de Pereira analizada desde la perspectiva de sistemas blandos. Congresos CLABES. Recuperado de https://revistas.utp.ac.pa/index.php/clabes/article/view/1567

Checkland, P. (2001). Systems thinking, systems practice (2. a ed.). Londres: Wiley.

Colmenares, A. M. (2012). Investigación-acción participativa: una metodología integradora del conocimiento y la acción. Voces y Silencios. Revista Latinoamericana de 


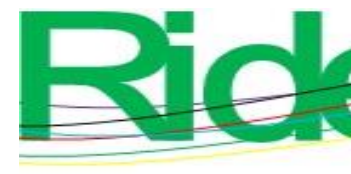

Educación, 3(1),
Revista Iberoamericana para la Investigación y el Desarrollo Educativo ISSN $2007-7467$

https://dialnet.unirioja.es/servlet/articulo?codigo $=4054232$

Consejo de Acreditación de la Enseñanza de la Ingeniería [CACEI] (2020). Marco de referencia 2020. Recuperado de http://cacei.org.mx/nvfs/nvfs02/nvfs0212.php

Devereux, G. (1994). De la ansiedad al método en las ciencias del comportamiento. México: Siglo XXI.

Emery, M. (2004). Open Systems Theory. In Boonstra, J. J. (ed.), Dynamics of Organizational Change and Learning (pp. 43-69). UK: John Wiley and Sons: Chichester. Doi: https://doi.org/10.1002/9780470753408.ch3

Esteve, J. M. (2003). Los modelos educativos de la sociedad contemporánea. En La tercera revolución educativa (pp. 123-151). España: Paidós.

Fleet, A. y González, P. (2017). Beca laboral universidad de Chile. Mecanismo de formación del talento y retención académica. Congresos CLABES. Recuperado de https://revistas.utp.ac.pa/index.php/clabes/article/view/1548.

Gibbs, P. (2017). Transdisciplinary higher education: a theoretical basis revealed in practice. Londres: Springer. Doi: 10.1007/978-3-319-56185-1

Glasserman, M. L. D., Reséndiz, C. M. y Riquelme, O, J. (2010). Aprendizaje orientado a proyectos como apoyo para la integración de asignaturas en la formación profesional. Apertura: Revista de Innovación Educativa, 2(2), 6-17. Recuperado de http://www.udgvirtual.udg.mx/apertura/index.php/apertura/article/view/137/152

Gracia, P. (2020). Autoconcepto físico, género y aprovechamiento escolar. Cambios en la transición a educación superior. En Roig-Vila, R. (coord.), Redes de investigación e innovación en docencia universitaria (pp. 995-1002). Alicante, Universidad de Alicante.

Güemes-Castorena, D. y Ponce-Jaramillo, E. (2019). University-Industry Linkage Framework to Identify Opportunity Areas. Review of Policy Research, 36, 660-682. Doi: https://doi.org/10.1111/ropr.12355

Hammersley, M. y Atkinson, P. (2007). Ethnography: Principles in practice. Londres: Routledge.

Hernández, S. R., Fernández, C. C. y Baptista, L. P. (2007). Fundamentos de metodología de la investigación. México: McGraw: Hill. 


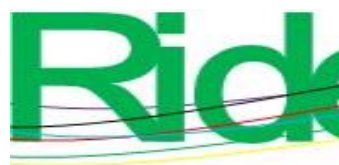

Revista Iberoamericana para la
Investigación y el Desarrollo Educativo
ISSN $2007-7467$

Herrero, V., Escanés, G. y Ayllón, S. (2017). Importancia de la actividad laboral al momento de ingreso en el abandono en distintas modalidades de cursado. Congresos CLABES. Recuperado de https://revistas.utp.ac.pa/index.php/clabes/article/view/1677

Hong, J., Zhu, R., Hou, B. and Wang, H. (2019). Academia-industry collaboration and regional innovation convergence in China. Knowledge Management Research \& Practice, 17(4), 396-407. Doi: 10.1080/14778238.2019.1589394

Ison, R. (2008). System thinking and practice for Action research. In Reason, P. and Bradbury, H. (eds.), Action research: Participative inquiry and practice (pp. 139158). Londres: Sage.

Ison, R. (2011). Cybersystemic conviviality: addressing the conundrum of ecosystems services. Cybernetics and Human Knowing, 18(1-2) pp. 135-141.

Ison, R. and Blackmore, C. (2014). Designing and developing a reflexive learning system for managing systemic change. Systems, 2(2), 119-136. Doi: $10.1108 / 03684920710827346$

Martínez, I. J. E., Tobón, S. y Romero S. A. (2017). Problemáticas relacionadas con la acreditación de la calidad de la educación superior en América Latina. Innovación Educativa, 17(73), 79-96. Recuperado de http://www.scielo.org.mx/scielo.php?script=sci_arttext\&pid=S166526732017000100079

McGhie, V. (2017). Entering university studies: identifying enabling factors for a successful transition from school to university. HigherEducation, 3(73), 407-422. Doi: $10.1007 / \mathrm{s} 10734-016-0100-2$

Munizaga, M. F., Cifuentes, O. M. y Beltrán, G. A. (2017). Variables y factores asociados al fenómeno de la retención y abandono estudiantil universitario en investigaciones de Latinoamérica y el Caribe. Congresos CLABES. Recuperado de http://revistas.utp.ac.pa/index.php/clabes/article/view/1671/2407

Press, C. U. (2015). Cambridge Dictionaries Online.

Rama, C. (2006). La tercera reforma de la educación superior en América Latina y el Caribe: masificación, regulaciones e internacionalización. Revista Educación y Pedagogía, 18(46), 11-24. Recuperado de http://www.ub.edu/histodidactica/images/documentos/pdf/ESuperior\%20en\%20AM ARICA\%20LATINA.pdf 


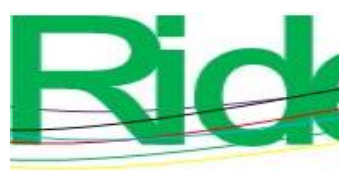

Revista Iberoamericana para la
Investigación y el Desarrollo Educativo
ISSN $2007-7467$

Reséndiz, C. M., Téllez, V. S. y Quintero, H. C. (2013). Estudio de requerimientos y especificaciones técnicas del sistema de impresión serigráfico para la industria gráfica del área de influencia a la UT. En Rojas, I. (ed.), Mejores prácticas de la gestión de procesos de manufactura, experiencias de investigación (pp. 60-66). Querétaro, México: Tercer Escalón. Recuperado de https://www.bibliotechnia.com.mx/portal/visor/web/visor.php

Reséndiz-Castro, M. (2019). Cibersistémica transdisciplinaria: intervención integral de tutoría. En Universidad Autónoma de San Luis Potosí (ed.), Innovación para la permanencia con equidad educativa: articulación institucional de programas en apoyo a la permanencia escolar (pp. 810-814). México: UASL- ANUIES.

Reséndiz C., M. (2021). Marco de trabajo cibersistémico transdisciplinario para mejorar el rendimiento académico de nivel educativo superior (tesis doctoral). SEPI ESIME Zacatenco IPN, México.

Reséndiz-Castro, M., Peón-Escalante, I. E. y Zepeda-Bautista, R. (2018). Aproximación metodológica cibersistémica transdisciplinaria al campo de la educación superior. En Sebastián, D. (coord.), XVII Congreso Nacional de Ingeniería Electromecánica y de Sistemas (CNIES 2018). México: IPN.

Rochin, B. F. L. (2021). Deserción escolar en la educación superior en México: revisión de literatura. RIDE, 11(22). Doi: https://doi.org/10.23913/ride.v11i22.821

Rodrigues, B. C. (1977). El método Paulo Freire para la alfabetización de adultos. Cuaderno del Centro de Cooperación Regional para la educación de adultos en América Latina y el Caribe (CREFAL 3). Recuperado de https://www.crefal.org/index.php?option=com_content $\&$ view=article $\&$ id=173\&Ite $\operatorname{mid}=225$

Romo, L. A. (2011). La tutoría. México: Anuies. Recuperado de http://publicaciones.anuies.mx/pdfs/libros/Libro225.pdf

Ronde, P. and Hussler, C. (2005). Innovation in regions: What does really matter? Research Policy, 34(8), 1150-1172. Doi: https://doi.org/10.1016/j.respol.2005.03.011

Said-Hung, E. (2017). Factores socioeducativos e institucionales asociados a la permanencia de los estudiantes de educación superior en línea caso UNIR-México. Congresos CLABES.

Recuperado de https://revistas.utp.ac.pa/index.php/clabes/article/view/1664 


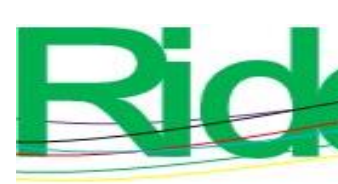

Revista Iberoamericana para la
Investigación y el Desarrollo Educativo
ISSN $2007-7467$

Singh, K. and Maloney, T. (2019) Using validated measures of high school academic achievement to predict university success. New Zealand Economic Papers, (53), 89106. Doi: https://doi.org/10.1080/00779954.2017.1419502

Stake, R. (1998). Investigación con estudio de casos. Madrid: Morata.

Strauss, A. y Corbin, J. (2002). Bases de la investigación cualitativa: técnicas y procedimientos para desarrollar la teoría fundamentada. Colombia: Universidad de Antioquia. Recuperado de https://diversidadlocal.files.wordpress.com/2012/09/bases-investigacioncualitativa.pdf

Tempelaar, D., Rienties, B., Mittelmeier, J. and Nguyen, Q. (2018). Student profiling in a dispositional learning analytics application using formative assessment. Computers in Human Behavior, 78. Doi: http://dx.doi.org/10.1016/j.chb.2017.08.010

Tobón, S. (2013). Formación integral y competencias. Pensamiento complejo, currículo, didáctica y evaluación (4. ${ }^{\mathrm{a}}$ ed.). Bogotá: ECOE. Recuperado de https://issuu.com/cife/docs/libro_formacion_integral_y_competen

Torres, G. J., Acevedo, C. D. y Gallo, G. L. (2016). Causas y consecuencias de la deserción y repitencia escolar: una visión general en el contexto latinoamericano. Cultura Educación $\quad y \quad$ Sociedad, 6(2). Recuperado de https://revistascientificas.cuc.edu.co/culturaeducacionysociedad/article/view/904 Valencia-Peris, A., Devís-Devís, J. and Peiró-Velert, C. (2016). Involvement in sedentary activities and academic performance in adolescents: Differences according to sociodemographic variables. Cultura y Educación, 28(2), 301-327. Doi: https://doi.org/10.1080/11356405.2016.1158451

Wiek, A., Ness, B., Schweizer-Ries, P., Brand, F. S. and Farioli, F. (2012). From complex systems analysis to transformational change: a comparative appraisal of sustainability science projects. Sustainability Science, 7(S1), 5-24. Doi: 10.1007/s11625-011-0148$\mathrm{y}$

Wilber, K. (2001). A brief history of everything. Boston, Massachusetts: Shambhala Publications.

Xing, W., Chen, X., Stein, J. and Marcinkowski, M. (2016). Temporal predication of dropouts in MOOCs: Reaching the low hanging fruit through stacking 


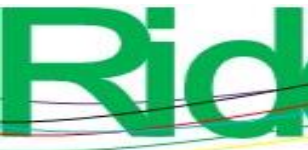

generalization. Computers

http://dx.doi.org/10.1016/j.chb.2015.12.007

Zabalza, B. M. A. y Lodeiro, E. L. (2019). El desafío de evaluar por competencias en la universidad. Revista Iberoamericana de Evaluación Educativa, 12(2), 29-47. Doi: 10.15366/riee2019.12.2

Zumárraga, M., Castro, M., Romero, J., Escobar, P., Boada, M., Armas, R., Luzuriaga, J. y Gonzáles, Y. (2017). Medición de intereses profesionales en estudiantes universitarios y un abordaje exploratorio de su relación con el desempeño académico. Congresos CLABES.

Recuperado

de https://revistas.utp.ac.pa/index.php/clabes/article/view/1621 


\begin{tabular}{|l|l|}
\hline Rol de Contribución & \multicolumn{1}{c}{$\begin{array}{c}\text { Revista Iberoamericana para la } \\
\text { Instin } 2007 \text { - } 7467\end{array}$} \\
\hline Conceptualización & Definición (solo poner nombre del autor) \\
\hline Metodología & Matilde Reséndiz-Castro \\
\hline Software & $\begin{array}{l}\text { Matilde Reséndiz-Castro (principal) Rosalba Zepeda-Bautista } \\
\text { (igual) }\end{array}$ \\
\hline Validación & (No aplica) \\
\hline Análisis Formal & $\begin{array}{l}\text { Matilde Reséndiz-Castro (principal) Rosalba Zepeda-Bautista } \\
\text { (apoya) }\end{array}$ \\
\hline Investigación & $\begin{array}{l}\text { Matilde Reséndiz-Castro (principal) Rosalba Zepeda-Bautista } \\
\text { (apoya) }\end{array}$ \\
\hline Recursos & $\begin{array}{l}\text { Matilde Reséndiz-Castro (principal) Rosalba Zepeda-Bautista } \\
\text { (igual) }\end{array}$ \\
\hline Curación de datos & $\begin{array}{l}\text { Matilde Reséndiz-Castro (principal) Rosalba Zepeda-Bautista } \\
\text { (igual) }\end{array}$ \\
\hline $\begin{array}{l}\text { Escritura - Preparación del } \\
\text { borrador original }\end{array}$ & $\begin{array}{l}\text { Matilde Reséndiz-Castro (principal) Rosalba Zepeda-Bautista } \\
\text { (apoya) }\end{array}$ \\
\hline $\begin{array}{l}\text { Escritura - Revisión y } \\
\text { edición }\end{array}$ & $\begin{array}{l}\text { Matilde Reséndiz-Castro } \\
\text { (apoya) }\end{array}$ \\
\hline Visualización & $\begin{array}{l}\text { Matilde Reséndiz-Castro (principal) Rosalba Zepeda-Bautista } \\
\text { (apoya) }\end{array}$ \\
\hline Supervisión & $\begin{array}{l}\text { Rosalba Zepeda-Bautista (principal) Matilde Reséndiz-Castro } \\
\text { (igual) }\end{array}$ \\
\hline Administración de Proyectos & $\begin{array}{l}\text { Matilde Reséndiz-Castro (principal) Rosalba Zepeda-Bautista } \\
\text { (igual) }\end{array}$ \\
\hline (igual) \\
\hline
\end{tabular}

